where $z_{1}(0)$, given and $z_{1}^{\prime}(0)=0$. The solution of this equation can be found in any standard text ${ }^{18}$ on differential equations, but, depending on the nature of $f(t)$, might best be done by numerical integration. In any event, we have $z(t)=z_{1}(t), 0 \leqq t \leqq 1$. Now during the second second we can again consider our system as a one degree of freedom system with the same mass, viscosity, and elastic coefficients, but with an impressed force which depends on $f(t)$ and velocity at time $t-1$. Consider the equation

$$
a z_{2}^{\prime \prime}(t)+b z_{2}^{\prime}(t)+c z(t)=f(t+1)-\beta z_{1}^{\prime}(t),
$$

for $0 \leqq t \leqq 1$, where $z_{2}(0)=z_{1}(1), z_{2}^{\prime}(0)=z_{1}^{\prime}(1)$, and $z_{1}^{\prime}(t)$ are derived from the solution of the previous equation. Again by standard methods we solve for $z_{2}(t), 0 \leqq t \leqq 1$. This is related to the actual response $z(t)$ during the second second by

$$
z(t)=z_{2}(t-1), \quad 1 \leqq t \leqq 2 .
$$

We can continue this process $^{19}$ for larger values of $t$ if so desired.

\title{
THE CENTER OF SHEAR AND THE CENTER OF TWIST*
}

\section{By A. WEINSTEIN (Carnegie Institute of Technology)}

In two recent papers W. R. Osgood ${ }^{1}$ and J. N. Goodier ${ }^{2}$ reconsider the much discussed question of the center of shear and center of twist, the former author pointing out the disagreement in the literature as to the location of the center of shear. However no mention is made of the important paper by $\mathrm{P}$. Cicala ${ }^{3}$ which, together with a paper of Trefftz, ${ }^{4}$ will form the basis of the following remarks.

R. V. Southwell ${ }^{5}$ has clearly pointed out that the two centers, which are intuitively well known to engineers, constitute two different concepts and are not just synonyms for the same point. The center of twist is the point at rest in every section of a uniform beam subject to a twist by a terminal couple and rigidly clamped at the other end. The center of shear (called also flexural center) is the point at which an applied shearing force would produce a flexure without torsion. However, neither of these points can be explicitly computed, since the displacements of a rigidly clamped beam under torsion are not known and, on the other hand, the concept of flexure without torsion is still to be exactly defined. Nevertheless Southwell, using Maxwell's reciprocal relations in a summary way, makes plausible the coincidence of both centers.

As Goodier points out, Saint Venant's theory of torsion and flexure of beams does

${ }^{18}$ E. L. Ince, Ordinary differential equations, Dover Publications, 1944, chap. 6.

${ }^{19}$ Analogous stepwise in tegration can be found in R. M. Head, Lag determination of altimeter systems, J. Aeronaut. Sci. 12, 85-93 (1945) and C. C. Kennedy, Measuring the Coulomb and viscous components of friction, Instruments 15, 404-410 (1942).

* Received May 9, 1946.

${ }^{1}$ W. R. Osgood, J. Appl. Mech. 10, A-62-A-64 (1943).

2 J. N. Goodier, J. Aeron. Sci. 11, 272-280 (1944).

${ }^{3}$ P. Cicala, Atti R. Acc. Sci. Torino 70, 356-371 (1935).

4 E. Trefftz, $Z$. angew. Math. Mech. 15, 220-225 (1935).

5 R. V. Southwell, An introduction to the theory of elasticity, 2nd ed., 1941, p. 29. 
not give any definition for either point. Several suggestions have been made concerning the use of Saint Venant's theory for this purpose, and it will be shown here that a definition can be given, which is based on Saint Venant's formulae and leads to an explicit proof of the coincidence of both centers.

We shall accept Trefftz' definition of the center of shear, and refer the reader to Trefftz' paper which also contains a clear exposition of the theory of flexure. It will suffice to mention here that Trefftz has been led to his definition by the reciprocity law of Maxwell and Betti.

Let $z$ be the axis of a uniform beam and $x, y$ the principal directions in the cross section $S$, the origin $O$ being the centroid of the cross section. According to Trefftz, the coordinates $x_{F}$ and $y_{F}$ of the center of shear are given by

$$
x_{F}=-\frac{1}{I_{x}} \iint_{S} y \varphi(x, y) d x d y, \quad y_{F}=\frac{1}{I_{y}} \iint_{S} x \varphi(x, y) d x d y,
$$

where $\varphi(x, y)$ denotes the warping function for the cross section in the theory of torsion and

$$
I_{x}=\iint_{S} y^{2} d x d y, \quad I_{y}=\iint_{S} x^{2} d x d y
$$

are the moments of inertia with respect to the axes of $x$ and $y$.

Turning now to the center of twist, the definition which will be given here will be based on an idea of Cicala, who however fails to recognize the coincidence of the two points as postulated by Southwell.

According to Saint-Venant, the displacements of the twisted beam are given by

$$
\begin{aligned}
u & =\tau(-z y+a+q z-r y), \\
v & =\tau(z x+b+r x-p z), \\
w & =\tau[\varphi(x, y)+c+p y-q x],
\end{aligned}
$$

where $\tau$ denotes the infinitesimal angle of twist. The last three terms in each formula represent a rigid body displacement. We have only six constants $a, b, c, p, q, r$ at our disposal, so that a rigid clamping cannot be obtained. However, an approximate clamping of the end section $z=0$ can be obtained by adopting the following postulates.

(i) The displacements $u$ and $v$ in the section $z=0$ are zero:

$$
u=0, \quad v=0 \text { for } z=0 .
$$

(ii) The mean square of the displacement $w$ in the $z$ direction is a minimum with respect to the parameters $c, p$ and $q$ which occur in the last Eq. (2):

$$
\iint_{S} w^{2} d x d y=\text { minimum. }
$$

Denoting this integral by $J$, we can write in place of (4)

$$
J(c, p, q)=\text { minimum with respect to } c, p \text { and } q \text {. }
$$

From (2) and (3) we obtain

$$
a=0, \quad b=0, \quad r=0,
$$


so that we have, for any section $z=$ const.,

$$
u=\tau z(q-y), \quad v=\tau z(x-p) .
$$

We see that, in every section, $u$ and $v$ vanish at the point $x=x_{T}, y=y_{T}$, where

$$
x_{T}=p, \quad y_{T}=q .
$$

This point will be, by definition, the center of twist. In order to compute $p$ and $q$, we use (4) or $\left(4^{\prime}\right)$, and set the derivatives of $J(c, p, q)$ with respect to $c, p$ and $q$ equal to zero. In this way we obtain the following equations.

$$
\iint_{S} w d x d y=0, \quad \iint_{S} w y d x d y=0, \quad \iint_{S} w x d x d y=0,
$$

Using the last Eqs. (2), namely $w=\tau(\varphi(x, y)+c+p y-q x)$, and observing that

$$
\iint_{S} y d x d y=\iint_{S} x d x d y=0,
$$

the origin being the centroid of the cross section, we obtain from (7) the three equations

$$
\iint_{S}(\varphi+c) d x d y=0, \quad \iint_{S}(\varphi+p y) y d x d y=0, \quad \iint_{S}(\varphi-q x) x d x d y=0 .
$$

The first of these equations yields the value of the constant $c$, while the second and the third gives us the coordinates $p=x_{T}$ and $q=y_{T}$ of the center of twist, namely

$$
x_{T}=-\frac{1}{I_{x}} \iint_{S} y \varphi(x, y) d x d y, \quad y_{T}=\frac{1}{I_{y}} \iint_{S} x \varphi(x, y) d x d y .
$$

A comparison with (1) shows that the center of shear coincides with the center of twist.

Incidentally, the first Eq. (7), namely $\iint_{S} w d x d y=0$, shows that the average displacement in the direction of the axis of the beam is zero.

It is interesting to note that both centers, as defined here, are independent of Poisson's ratio. 